\title{
Tumor Embolism
}

National Cancer Institute

\section{Source}

National Cancer Institute. Tumor Embolism. NCI Thesaurus. Code C3005.

A tumor embolism involves sudden blockage of an artery by a clot or by other material from tumor fragments transported by the blood stream. 